\title{
The Vortex Method With Finite Elements
}

\author{
By Claude Bardos, Michel Bercovier and Olivier Pironneau
}

\begin{abstract}
This work shows that the method of charcteristics is well suited for the numerical solution of first order hyperbolic partial differential equations whose coefficients are approximated by functions piecewise constant on a finite element triangulation of the domain of integration. We apply this method to the numerical solution of Euler's equation and prove convergence when the time step and the mesh size tend to zero. The proof is based upon the results of regularity given by Kato and Wolibner and on $L^{\infty}$ estimates for the solution of the Dirichlet problem given by Nitsche. The method obtained belongs to the family of vortex methods usually studied in a finite difference context.
\end{abstract}

Introduction. The vortex method is based on an old concept of fluid mechanics which says that for two-dimensional nonviscous flows the vorticity in the fluid is transported by the flow; thus, if the initial distribution of vorticity consists of a finite number of point vortices, the flow at later times can be found by transport of these point vortices along the streamlines of the flow that they create.

In mathematical terms this means that the two-dimensional stream functionvorticity formulation of Euler's equations

$$
\left\{\begin{array}{l}
\left.\frac{\partial \omega}{\partial t}+u \nabla \omega=0, \quad-\Delta \Psi=\omega, \quad u=\nabla \Lambda \Psi \text { in } \Omega \times\right] 0, T[, \\
\omega(t=0)=\Sigma \omega_{i}^{0} \delta\left(x-x_{i}\right),\left.\quad \Psi\right|_{\Gamma}=\Psi_{0},
\end{array}\right.
$$

where $\delta$ is the Dirac function, $\Gamma$, the boundary of $\Omega$, is integrated by

$$
\left\{\begin{array}{l}
\omega(x, t)=\sum \omega_{i}^{0}\left(x-x_{i}(t)\right), \\
\frac{d x_{i}}{d t}=\nabla \Lambda \Psi\left(x_{i}, t\right), \quad x_{i}(t=0)=x_{i}, \\
-\Delta \Psi=\sum \omega_{i}^{0} \delta\left(x-x_{i}(t)\right),\left.\quad \Psi\right|_{\Gamma}=\Psi_{0} .
\end{array}\right.
$$

This method was first implemented by Christiansen [6] and Chorin [5] and thoroughly tested by Baker [2] on the roll up of vortex sheets. From the theoretical point of view if $\Omega=R^{2}$ Hald [10] showed that when (2) is discretized explicitly in time, when the Dirichlet problem is approximated by a suitable discretization of the corresponding Green's function and when the Dirac functions are smoothed by appropriate convolutions then the method converges. In Baker [2] the Dirichlet problem is discretized with finite differences, and as far as we know the convergence is not established in such a case.

The present work is based on the rather straightforward observation that the system (2) is perhaps easier to analyze when it is discretized by the finite element

Received May 1, 1980; revised June 30, 1980.

1980 Mathematics Subject Classification. Primary 65N30, 65N35; Secondary 76C05. 
method than by the two previously mentioned methods because the equation for the characteristics $x_{i}(t)$ can be integrated exactly if $\nabla \Lambda \Psi$ is piecewise constant on a triangulation of $\Omega \times] 0, T$. However, the error analysis shows that in the finite element context it is no longer feasible to work with Dirac functions; it is better to use a piecewise constant discretization of $\omega^{0}(x)$. Therefore, we shall not work with point vortices but with a piecewise constant approximation of the vortex field

$$
\omega(x, t)=\sum_{i=1}^{N} \omega_{j(i)}^{0} I\left(x-x_{i}(t)\right)
$$

where $I\left(x-x_{i}(t)\right)$ equals 1 if $x$ and $x_{i}(t)$ belong to the same element of the triangulation and zero otherwise, where $N$ is the total number of elements and where $j(i)$ is the index of the element to which $x_{i}(0)$ belongs. Therefore we will have to compute certain characteristics backward in $t$ in order to define $\omega(x, t)$ by (3).

Thus, although in spirit identical, in practice the present method is substantially different from the point vortex method of [2] and [5]. Both have the advantage of being nondissipative; ours is conservative in a statistical sense only in terms of $\omega^{0}$. On the other hand, we do not have to insert new vortices in some regions of the flow as in [6] and the method is more appropriate to smooth flows. But most of all an error analysis will be given and the method is unconditionally stable in time. This, by the way, may also be true of the cloud and cell vortex method [5], [2] as was observed by Baker.

The proofs are involved and difficult in their details but the guidelines are simple: we assume that the regularity obtained by Wolibner [14] and Kato [11] for the solution holds. Thus, to measure the error between the exact solution and the appropriate solution we measure the distance between two particles, one transported by the exact flow and the other by the approximate flow. In the process $L^{\infty}$ estimates of the finite element solution of the Dirichlet problem for $-\Delta$ will have to be established following the arguments of Nitsche [12].

For the sake of clarity and also because the method of characteristics in the finite element context can be useful for other hyperbolic systems, we begin with a presentation of the method for the transport equation. Then, in Section 2 the method and the error analysis is explained for the two-dimensional Euler equation.

Finally, the numerical implementation and some numerical tests are presented in Section 3.

\section{Finite Elements and Characteristics for the Transport Equation.}

1. a. Statement of the Problem. Let $\Omega$ be a bounded open set of $R^{n}$, let $Q=$ $Q \times] 0, T\left[\right.$, and $u$ a divergence free $(\nabla \cdot u=0)$ vector of $\left(H^{1}(Q) \cap L^{\infty}(Q)\right)^{n}$. Let $f$ and $\rho_{0}$ be two functions of $L^{\infty}(Q)$ and consider the problem

$$
\begin{aligned}
& \left.\frac{\partial \rho}{\partial t}+u \nabla \rho=f \text { in } \Omega \times\right] 0, T[=Q, \\
& \rho(x, t)=\rho_{0}(x, t) \text { for all }\{x, t\} \in S=\Omega \times\{0\} \cup \Sigma^{-},
\end{aligned}
$$

where $\Gamma$ is the boundary of $\Omega, \nu$ is outward normal and $\Sigma^{-}=\{\{x, t\}: u(x, t) \cdot \nu \leqslant$ $0, x \in \Gamma\}$.

$\Sigma^{-}$represents the part of the boundary of $Q$ where the flow enters into $\Omega$; thus 
the boundary conditions for $\rho$ are given at initial time and when the velocity $u$ enters into $\Omega$.

Several physical phenomena are governed by this equation, known as the transport equation. On rectangular domains $\Omega(1.1)$ is easily discretized by any upwind finite difference scheme, but if $\Omega$ is complicated there is no simple nondissipative finite element scheme, and the method of characteristics is usually considered as an expensive numerical method. Let us show that for first order accurate discretizations this is not so costly.

The method of characteristics is based upon the following observation: given $\{x, t\} \in Q$ define $\left\{X^{x, t}(\tau), \tau\right\}$ by

$$
\frac{d X}{d \tau}= \begin{cases}u(X(\tau), \tau) & \text { if } X(\tau) \in \Omega, \\ 0 \text { if } X(\tau) \notin \Omega, & \forall \tau \in] 0, t[, \\ X(t)=x . & \end{cases}
$$

If $u$ is uniformly Lipschitz continuous with respect to $x,(1.2)$ has a unique solution on $] 0, t[$; then we define

$$
\hat{\rho}(x, t)=\rho_{0}\left(X^{x, t}(0), 0\right)+\int_{0}^{t} f\left(X^{x, t}(\tau), \tau\right) d \tau
$$

and claim that $\hat{\rho}$ is a solution of (1.1). If the data $u, \rho_{0}$ and $f$ are not smooth the proof is difficult [15], but if $\rho_{0}, f$ and $u$ are in $C^{1}(Q)$, then $\hat{\rho}$ is differentiable with respect to $\{x, t\}$ and

$$
\begin{aligned}
\hat{\rho}\left(X^{x, t}(\tau), \tau\right)-\hat{\rho}(x, t)= & \frac{\partial \hat{\rho}}{\partial t}(\tau-t)+\nabla_{x} \hat{\rho}\left(X^{x, t}(\tau)-x\right) \\
& +o(\tau-t)+o(X-x) \\
= & \left(\frac{\partial \hat{\rho}}{\partial t}+u \nabla_{x} \hat{\rho}\right)(\tau-t)+o(\tau-t)
\end{aligned}
$$

and also

$$
\hat{\rho}\left(X^{x, t}(\tau), \tau\right)-\hat{\rho}(x, t)=\int_{t}^{\tau} f\left(X^{x, t}(\sigma), \sigma\right) d \sigma .
$$

Therefore we have the following result:

Proposition 1. When $u$ is uniformly Lipschitz continuous in $x$ and divergence free and $\rho_{0}, u$ and $f$ are in $L^{\infty}(Q)$, then the solution of (1.1) is given by (1.2), (1.3).

Remark. Equation (1.2) includes the possibility that a characteristic leaves $\Omega$ before $t=0$ with the convention that $\left.f\right|_{\Gamma}=0$.

1.b. Discretization. To discretize (1.1) we choose a triangulation $\mathcal{T}_{h}$ of $\Omega$ made of nonoverlapping triangles if $n=2$ or tetrahedra if $n=3$ with the usual properties [4]:

$$
\mathcal{T}_{h}=\left\{\tau_{i}\right\}_{i=1}^{N},
$$

$\tau_{i} \cap \tau_{j}=\varnothing$ or one vertex, or one side (or face), (or one edge),

$$
\bigcup_{1}^{N} \tau_{1}=\Omega_{h} \subset \Omega \text {, distance }\left(\Omega_{h}, \Omega\right)=O\left(h^{2}\right), h=\text { size of largest side of } \mathscr{T}_{h} \text {. }
$$


If $\Delta t$ denotes a time step then $Q$ is approximated by

$$
\left.Q_{h}=\bigcup P_{i j} ; \quad P_{i j}=\tau_{i} \times\right] j \Delta t,(j+1) \Delta t[, \quad j=1, \ldots, M=E(T / \Delta t) .
$$

Instead of $u$ we shall approximate the stream function $\Psi$ of $u$, i.e. the function such that

$$
\begin{aligned}
u & =\nabla \Lambda \Psi=\left(\frac{\partial \Psi_{3}}{\partial x_{2}}-\frac{\partial \Psi_{2}}{\partial x_{3}}, \frac{\partial \Psi_{1}}{\partial x_{3}}-\frac{\partial \Psi_{3}}{\partial x_{1}}, \frac{\partial \Psi_{2}}{\partial x_{1}}-\frac{\partial \Psi_{1}}{\partial x_{2}}\right) \text { if } n=3 \\
& =\left(\frac{\partial \Psi}{\partial x_{2}},-\frac{\partial \Psi}{\partial x_{1}}\right) \quad \text { if } n=2
\end{aligned}
$$

Let $\Psi_{h}$ be an approximation of $\Psi$ in the space $H_{h}$ :

$$
H_{h}=\left\{\varphi_{h}: \varphi_{h} \text { is continuous in } x ;\left.\nabla \varphi_{h}\right|_{P_{i j}} \text { is linear in } x \text { and constant in } t, \forall i j\right\} \text {. }
$$

Then we approximate $u$ by a function which is constant on each $P_{i j}$ :

$$
u_{h}=\nabla \Lambda \Psi_{h}
$$

and we shall denote by $\rho^{h}(x, t)$ the soluton of

$$
\begin{aligned}
& \left.\frac{\partial \rho^{h}}{\partial t}+u_{h} \nabla \rho^{h}=f_{h} \quad \text { in } \Omega_{h} \times\right] 0, T[, \\
& \rho^{h}(x, t)=\rho_{0 h}(x, t) \quad \text { on } S_{h},
\end{aligned}
$$

where $f_{h}$ and $\rho_{0 h}$ are piecewise constant approximations of $f$ and $\rho_{0}$.

Even though $\rho^{h}$, given by (1.10), belongs to a finite-dimensional function space we approximate it further by choosing a point $\left\{\xi^{i, j}, t^{j}\right\}$ in each $P_{i j}$ and set

$$
\rho_{h}(x, t)=\rho^{h}\left(\xi^{i j}, t^{j}\right) \quad \forall\{x, t\} \in P_{i j} \forall i=1, \ldots, N, j=1, \ldots, M .
$$

Proposition 2. For almost all choices of $\left\{\xi^{i j}, t^{j}\right\} \in P_{i j}, \rho_{h}$ is uniquely defined by (1.10), (1.11) and computable in a finite number of operations by the following algorithm.

Algorithm 1: Computes the solution of (1.10) for one $x$ and $t$.

1. Find the prism $P_{i j}$ which contains $\{x, t\}$; set $\left\{x^{0}, t^{0}\right\}=\{x, t\}$ and $m=0$.

2. Compute $\lambda \in R^{+}$such that $\left\{x^{m}-\lambda u_{h}\left(x^{m}, t^{m}\right), t^{m}-\lambda\right\} \in \partial P_{i j}$, the boundary of $P_{i j}$.

3. Set $x^{m+1}=x^{m}-\lambda u_{h}\left(x^{m}, t^{m}\right), t^{m+1}=t^{m}-\lambda$. If $t^{m+1}=0$ or $x^{m+1} \in \partial \Omega_{h}$ go to 4 , else find $P_{k l}$ such that

$$
\left\{x^{m+1}, t^{m+1}\right\} \in P_{k l},\left.\quad u_{h}\left(x^{m+1}, t^{m+1}\right)\right|_{P_{k l}} \text { points inside } P_{k l},
$$

and go back to 2 with $m=m+1, i=k, j=l$.

4. Set $\rho^{h}(x, t)=\rho_{0 h}\left(x^{m+1}, t^{m+1}\right)+\sum_{i=0}^{m} f_{h}\left(x^{i}, t^{i}\right)\left(t^{i+1}-t^{i}\right)$.

Proof. Since $u_{h}$ is piecewise constant on $Q_{h}$, the characteristic that passes through a given point $\{x, t\}$ is a broken straight line, which is determined by its nodes. These are located at the discontinuities of $u_{h}$, therefore on the boundaries of the $P_{i j}$.

Condition (1.9) insures that a $P_{k l}$ satisfying the condition of step 3 can be found. In fact in the sense of distributions $u_{h}$ is divergence free. Therefore, the normal components of $u_{h}$ are continuous across the sides of the triangles thus (1.12) can be fulfilled. However, this procedure does not give a unique solution whenever the 
broken straight line passes through a vertex. This is why Proposition 2 is stated for almost all $\left\{\xi_{j}, t_{j}\right\}$.

Algorithm 1 simply states that the characteristic to be computed can be found by starting from $\{x, t\}$ and following the directions $-u_{h}$ from one $P_{i j}$ to the next.

Remark. Construction (1.9) may prove to be expensive because it costs a Dirichlet problem at each time step. In most cases $u_{h}$ can be discretized directly but then the algorithm may get stuck in one element.

1.c. Implementation. In practice one usually computes $\rho_{h}$ for all $\{x, t\} \in Q_{h}$ : therefore it would be too costly to compute $N \times M$ characteristics. It is more feasible to proceed as follows: Pick one point in each element and compute the characteristic that begins here forward in time. If at some time step an element is not crossed by one of these characteristics, pick a new point inside the element and compute the characteristic backward in time that ends at this new point. At each time step make sure that no more than $N$ characteristics are stored, i.e. discard some characteristics if they end up in the same element. This gives the following algorithm:

Algorithm 2: Computes $\rho_{h}(x, t)$ solution of (1.10)-(1.11) for all $\{x, t\} \in Q_{h}$.

0 . Choose $\xi^{i 0} \in \tau_{i}, i=1, \ldots, N$. Set $t^{0}=0, j=0$.

1. For all $i=1$, to $M$ do

with Procedure $F\left(\xi^{i j}, t^{j}, \Delta t, u_{h}, 1\right)$ compute all the nodes $\left\{x_{m}^{i j}, t_{m}^{i j}\right\}_{m=1}^{m_{i j}}$ of the characteristic solution of

$$
\frac{d x}{d \tau}=\left\{\begin{array}{l}
u_{h}(x, \tau) \text { if } x \in Q_{h}, \\
0 \text { otherwise, }
\end{array}\right.
$$

for all $\left.\tau \in] t^{j}, t^{j}+\Delta t\right]$ with $X\left(t^{j}\right)=\xi^{i j}$.

Set

$$
\begin{gathered}
\xi^{k(i) j+1}=x_{m_{i}}^{i j}, t^{j+1}=t^{j}+\Delta t \\
\rho_{h}\left(\xi^{k(i) j+1}, t^{j+1}\right)=\rho_{h}\left(\xi^{i j}, t^{j}\right)+\sum_{1}^{m_{i j}-1} f_{h}\left(x_{m}^{i j}, t_{m}^{i j}\right)\left(t_{m+1}^{i j}-t_{m}^{i j}\right)
\end{gathered}
$$

where $k(i)$ is such that

$$
\xi^{k(i) j+1} \in P_{k(i) j+1}
$$

2. For all $i^{\prime} \leqslant N$ such that there are no $k(i)=i^{\prime}$ with Procedure $F\left(\xi^{i^{\prime} 0}, t^{j+1}, t^{j+1}-t^{j-1},-u_{h},-1\right)$ compute all the nodes $\left\{x_{m}^{i j}, t_{m}^{i j}\right\}_{1}^{m_{i j}}$ of the characteristic solution of (1.13) for all $\tau \in] 0, t^{j+1}$ ] with $X\left(t^{j+1}\right)=\xi^{i^{\prime} 0}$, and set

$$
\begin{gathered}
\xi^{i^{\prime} j+1}=\xi^{i^{\prime} 0} \\
\rho_{h}\left(\xi^{i^{\prime} j+1}, t^{j+1}\right)=\rho_{0 h}(X(0))+\sum_{1}^{m_{i j}} f_{h}\left(x_{m}^{i j}, t_{m}^{i j}\right)\left(t_{m}-t_{m+1}\right) .
\end{gathered}
$$

3. Replace $j$ by $j+1$ and stop if $j>M$, else keep only one $\xi^{i j}$ per $\tau_{i}$ and go back to 1 .

Remark. The nodes $\left\{x_{m}^{i j}, t_{m}^{i}\right\}$ of the characteristics are the vertices of these broken lines. 
Procedure $F\left(\xi, \tau, \Delta \tau, v_{h}, \varepsilon\right)$. Computes with the method of Algorithm 1 the intersections $\left\{x_{m}, t_{m}\right\}$ with the boundaries $\partial P_{i j}$ of the elements of the triangulation of $Q_{h}$, of the characteristic $X(t)$ solution of

$$
\frac{d X}{d t}=v_{h}(X, t), \text { or } 0 \text { if } X \notin \Omega_{h} \begin{cases}\text { on }] \tau, \tau+\Delta \tau] & \text { if } \varepsilon=+1, \\ \text { on }[\tau-\Delta \tau, \tau[ & \text { if } \varepsilon=-1,\end{cases}
$$

with $X(\tau)=\xi$.

Alternatively we may choose to use

Algorithm 3: Computes the solution $\rho_{h}(x, t)$ of (1.10), (1.11).

0 . Choose $\theta=T / r, r \in \mathbf{N}$.

1. For $i=1$ to $r$ compute with Algorithm 2 the solution of (1.10), (1.11), over $t \in\left[(i-1) \theta, i \theta\left[\right.\right.$ with $\rho_{h}(\cdot,(i-1) \theta)$ for initial condition.

Comments. For regular fields $u_{h}$ most elements are expected to contain one end point of the characteristics that are computed forward in time. For those elements which have no such characteristic at a given time we pick any point, here $\xi^{i 0}$, and compute the characteristic that ends at $\xi^{i 0}$, backward in time; last, note that it is not absolutely necessary to go back till $t=0$; the user may pick a time $\theta$ and $\rho_{h}(t)$ can be computed from $\rho_{h}(t-\theta)$ according to (1.18); this gives Algorithm 3. These schemes could be made conservative by putting appropriate weights in (1.14), (1.15) according to the number of characteristics that end in the same element and start from the same element, but the error analysis below would no longer be valid.

The number of operations, $N X$, of Algorithm 3 is of the order of

$$
N X \leqslant\left(\frac{D^{2} T}{h^{2} \Delta t} \max \left(\frac{u_{h} \Delta t}{h}, 1\right)+\nu \frac{u_{h} \theta}{\Delta t}\right) C_{1}
$$

where $C_{1}$ is some constant of the order of $10, \nu$ is an upper bound on the number of backward characteristics computed at each time step, $D$ is the diameter of $\Omega_{h}$.

We have found that the best choice for $\Delta t$ is the one that makes the characteristics cross one element, i.e.,

$$
\Delta t \simeq h / u_{h}
$$

Then even for $\theta=T$, assuming that $\nu$ is of the order of $D / h$, the method is $O\left(1 / h^{3}\right)$, that is, comparable to the work necessary to solve a Dirichlet problem with a good finite element method.

1.d. Error Analysis. We shall denote by $|\cdot|_{p, \Omega}$ the $L^{p}(\Omega)$ norm. For convenience we assume that $u \cdot n=0$ on $\Gamma$, in order to derive the error estimates in this section.

Theorem 1. If $\rho_{h}$ is computed by Algorithm 2 then the $L^{\infty}$ error is $O(h+\Delta t)$ for smooth data. More precisely

$$
\begin{aligned}
& \left|\rho_{h}(\cdot, t)-\rho(\cdot, t)\right|_{\infty, \Omega} \\
& \quad \leqslant T\left|f_{h}-f\right|_{\infty, Q}+\left|\rho_{0 h}-\rho_{0}\right|_{\infty, \Omega}+C_{2}\left|\nabla \Lambda \Psi_{h}-\nabla \Lambda \Psi\right|_{\infty, Q}
\end{aligned}
$$


Proof. Let $X_{h}^{x, t}$ be a characteristic computed by Algorithm 1. Then

$$
\begin{aligned}
\left|\rho^{h}(x, t)-\rho(x, t)\right| \leqslant & \int_{0}^{t}\left|f_{h}\left(X_{h}^{x, t}(\tau), \tau\right)-f\left(X^{x, t}(\tau), \tau\right)\right| d \tau \\
& +\left|\rho_{0 h}\left(X^{x, t}(0)\right)-\rho_{0}\left(X_{h}^{x, t}(0)\right)\right| \\
\leqslant & |\nabla f|_{\infty, Q} \int_{0}^{t}\left|X_{h}^{x, t}(\tau)-X^{x, t}(\tau)\right| d \tau+\left|f_{h}-f\right|_{1, Q} \\
& +\left|\nabla \rho_{0}\right|_{\infty, \Omega}\left|X_{h}^{x, t}(0)-X^{x, t}(0)\right|+\left|\rho_{0 h}-\rho_{0}\right|_{\infty, \Omega}
\end{aligned}
$$

On the other hand

$$
\delta(\tau)=X_{h}^{x, t}(\tau)-X^{x, t}(\tau)
$$

satisfies

$$
|\dot{\delta}|=\left|\nabla \Lambda \Psi_{h}\left(X_{h}, \tau\right)-\nabla \Lambda \Psi(x, \tau)\right| \leqslant\left|\nabla \Lambda \Psi_{h}-\nabla \Lambda \Psi\right|_{\infty, Q}+\left|\Psi^{\prime \prime}\right|_{\infty, Q}|\delta|,
$$

and from the Bellman-Gronwall lemma this yields

$$
|\delta(\tau)| \leqslant \frac{\left|\nabla \Lambda \Psi_{h}-\nabla \Lambda \Psi\right|_{\infty, Q}}{\left|\Psi^{\prime \prime}\right|_{\infty, Q}}\left(-1+\exp \left(\left|\Psi^{\prime \prime}\right|_{\infty, Q}(t-\tau)\right)\right) .
$$

Therefore

$$
\begin{aligned}
& \left|\rho^{h}(x, t)-\rho(x, t)\right| \\
& \quad \leqslant T\left|f_{h}-f\right|_{\infty, Q}+\left|\rho_{0 h}-\rho_{0}\right|_{\infty, \Omega}+C_{2}\left|\nabla \Lambda \Psi_{h}-\nabla \Lambda \Psi\right|_{\infty, Q} .
\end{aligned}
$$

Now we make the same analysis with the characteristics computed forward in time and we get the result.

The fact that (1.22) implies an error of order $h$ and $\Delta t$ is classical (see Ciarlet [4], for example).

Remark. Of course in many applications $\rho$ is not in $W^{1, \infty}(\Omega)$ and therefore the error estimate (1.22) does not hold. For example (1.22) does not hold in case of shocks; it only gives an idea of the precision of the method locally in a region where the solution is smooth.

It may prove to be too memory-consuming to take $\theta=T$.

TheOREM 2. For smooth data Algorithm 3 yields also

$$
\left|\rho_{h}(\cdot, t)-\rho(\cdot, t)\right|_{\infty, \Omega} \leqslant C_{3}\left(\frac{h}{p \Delta t}+\Delta t\right) \quad \forall t, \forall \theta=p \Delta t, p>1 .
$$

Proof. As for Theorem 1

$$
\begin{aligned}
\varepsilon_{h}^{j}= & \left|\rho_{h}\left(\xi^{i j}, j \Delta t\right)-\rho\left(\xi^{i j}, j \Delta t\right)\right| \\
\leqslant & \left|\rho_{h}\left(X_{h}^{\xi^{i j} j \Delta t}((j-p) \Delta t)\right)-\rho\left(X^{\xi^{i j} j \Delta t}((j-p) \Delta t)\right)\right| \\
& +\int_{(j-p) \Delta t}^{j \Delta t}\left|f_{h}\left(X_{h}^{\xi^{i j} j \Delta t}(\tau), \tau\right)-f\left(X^{\xi^{i j} j \Delta t}(\tau), \tau\right)\right| d \tau \\
\leqslant & \left|\rho_{h}(\cdot,(j-p) \Delta t)-\rho(\cdot,(j-p) \Delta t)\right|_{\infty} \\
& +|\nabla \rho|_{\infty}\left|X_{h}^{\xi^{i j} j \Delta t}((j-p) \Delta t)-X^{\xi^{i j} j \Delta t}((j-p) \Delta t)\right| \\
& +\left|f-f_{h}\right|_{\infty} p \Delta t+|\nabla f|_{\infty} \int_{(j-p) \Delta t}^{j \Delta t}\left|X_{h}^{\xi_{i j}, \Delta t}(\tau)-X^{\xi_{i j} j \Delta t}(\tau)\right| d \tau
\end{aligned}
$$


Now we make use of (1.26) with $\tau=(j-p) \Delta t$ and $t=j \Delta t$ to find that

$$
\begin{aligned}
\varepsilon_{h}^{j \leqslant \varepsilon_{h}^{j-p}} & +\left(|\nabla \rho|_{\infty}+\Delta t p|\nabla f|_{\infty}\right) \frac{\left|\nabla \Lambda \Psi_{h}-\nabla \Lambda \Psi\right|_{\infty, Q}}{\left|\Psi^{\prime \prime}\right|_{\infty, Q}}\left(\exp \left(\left|\Psi^{\prime \prime}\right|_{\infty, Q} p \Delta t\right)-1\right) \\
& +\left|f-f_{h}\right|_{\infty} p \Delta t+|\nabla \rho|_{\infty} h .
\end{aligned}
$$

It remains to sum up the above inequalitites from $j=t / \Delta t$ to $j=p$; it yields

$$
\begin{aligned}
\left|\rho_{h}(\cdot, t)-\rho(\cdot, t)\right|_{\infty, \Omega} \leqslant & |\nabla \rho|_{\infty}(h+\Delta t)+\left|\rho_{0 h}-\rho_{0}\right|_{\infty}+|\nabla \rho|_{\infty} h / p \Delta t \\
& +\left|f-f_{h}\right|_{\infty} T\left(p \Delta t|\nabla f|_{\infty}+|\nabla \rho|_{\infty}\right) \\
& \cdot\left|\nabla \Lambda \Psi_{h}-\nabla \Lambda \Psi\right|_{\infty, Q} T \frac{\exp \left(\left|\Psi^{\prime \prime}\right|_{\infty, Q} p \Delta t\right)-1}{\left|\Psi^{\prime \prime}\right|_{\infty, Q} p} .
\end{aligned}
$$

Remark. Thus, from the point of view of error estimates, it does not seem necessary to go back very far in time along the characteristics to compute $\rho_{h}$. From the point of view of numerical practice, however, there is much less dissipation when $\theta$ is large.

It is interesting to note that the present framework is well suited to show the convergence of the classical method of characteristics. To obtain a scheme that is $O(h+\Delta t)$, it is sufficient to use Euler's method to compute $X_{h}^{x, t}(\cdot)$ :

$$
X_{h}^{x, t}(t-\Delta t)=x-u_{h}(x, t) \Delta t .
$$

However, $\rho_{h}$ must be interpolated from its values at the vertices of $\mathcal{T}_{h}$, piecewise linearly in $x$. Also $X_{h}$ might not be in $\Omega_{h}$, thus $u_{h}$ must be extended outside $\Omega_{h}$. Again for simplicity but without loss of generality we assume $\left.u \cdot n\right|_{\Gamma}=0$.

Proposition 3. Under the above assumptions, if $\rho_{h}(\cdot, t)$ is piecewise linear on $\mathfrak{T}_{h}$ and $\forall x$ vertex of $\left.\mathcal{T}_{h}, \forall t \in\right](j-1) \Delta t, j \Delta t[$

$$
\rho_{h}(x, t)=\rho_{h}\left(X_{h}^{x, t^{j}}\left(t^{j-1}\right), t^{j-1}\right)+f_{h}(x, t) \Delta t
$$

where $X_{h}$ is computed by (1.29) then for smooth data ( $u$ and its extension and $f$ in $L^{\infty}(Q), \rho(\cdot, t)$ in $\left.W^{1, \infty}(\Omega)\right)$

$$
\left|\rho_{h}-\rho\right|_{\infty} \leqslant c\left(h+\Delta t+h^{2} / \Delta t\right)
$$

Proof. We proceed as before:

$$
\begin{aligned}
\mid \rho_{h}(\xi, j \Delta t)-\rho(\xi, j \Delta t) & |\leqslant| \rho_{h}(\cdot,(j-1) \Delta t)-\left.\rho(\cdot,(j-1) \Delta t)\right|_{\infty} \\
+ & |\nabla f|_{\infty} \int_{(j-1) \Delta t}^{j \Delta t}\left|X_{h}^{\xi j \Delta t}(\tau)-X^{\xi j \Delta t}(\tau)\right| d \tau \\
+ & |\nabla \rho|_{\infty}\left|X_{h}^{\xi, j t}((j-1) \Delta t)-X^{\xi j \Delta t}((j-1) \Delta t)\right|+\left|f_{h}-f\right|_{\infty} \Delta t .
\end{aligned}
$$

From (1.29) we get

$$
\left|X_{h}^{\xi, j \Delta t}((j-1) \Delta t)-X^{\xi, j \Delta t}((j-1))\right| \leqslant\left|u_{h}-u\right|_{\infty} \Delta t .
$$

which yields the result, because

$$
\left|\rho_{h}(\cdot, j \Delta t)-\rho(\cdot, j \Delta t)\right|_{\infty} \leqslant \max _{\xi \text { vertex }}\left|\rho_{h}(\xi, j \Delta t)-\rho(\xi, j \Delta t)\right|+|\nabla(\nabla \rho)|_{\infty} h^{2} .
$$

Remark. From the point of view of error analysis it is not necessary to compute the characteristics with the accuracy chosen but from the numerical point of view (1.29) (as well as $\theta=\Delta t$ in Algorithm 3) introduces a large discretization error. 
Theoretically the method is nondissipative; for example if $\Omega$ is a duct and $\rho_{0}=1$ for $x<0$ and $\rho_{0}=0$ for $x>0$ and if $u=(1,0)$ then $\rho_{h}$ can take only the values 1 or 0 . But nevertheless the method will distort the shock, so that in a statistical sense it is dissipative. Let us give a heuristic argument to estimate the statistical dissipativity of the method.

Equation (1.10) can be viewed as

$$
\begin{gathered}
\left.\frac{\partial \rho^{h}}{\partial t}+\left(u+\eta_{h}\right) \nabla \rho^{h}=f+\eta_{h}^{\prime} \quad \text { in } \Omega_{h} \times\right] 0, T[ \\
\rho^{h}(x, t)=\rho_{0}(x, t)+\eta_{h}^{\prime \prime}(x, t) \text { on } S_{h}^{-},
\end{gathered}
$$

where $\eta_{h}, \eta_{h}^{\prime}, \eta_{h}^{\prime \prime}$ are the errors due to the discretization.

Assume that each triangulation is taken with a given probability; then the errors become random variables and $\rho^{h}$ can be seen as a random process solution of a stochastic partial differential equation.

It is known that if $\eta, \eta^{\prime}, \eta^{\prime \prime}$ are Gaussian processes with zero mean then the expected value $\bar{\rho}$ of $\rho^{h}$ will satisfy

$$
\left.\frac{\partial \bar{\rho}}{\partial t}+u \nabla \bar{\rho}-\nabla \cdot(\sigma \nabla \bar{\rho})=f \quad \text { in } \Omega \times\right] 0, T\left[, \quad \bar{\rho}=\rho_{0} \quad \text { on } S^{-},\right.
$$

where $\sigma$ is the variance of $\eta_{h}$.

In our case of course it may be difficult to show that $\eta_{h}$ is Gaussian when the triangulations $\mathcal{T}_{h}$ are chosen with equal probability, but if it were then $\sigma$ would be proportional to $h^{2}$, so that in all likelihood there exists $C_{4}$ such that $\bar{\rho}$ satisfies (1.31) with $\sigma=C_{4} h^{2}$. Then we may say that statistically the order of dissipation is $O\left(h^{2}\right)$.

\section{Application to the Euler Equation in Two Dimensions: Construction of the approximate Solution and Error Bounds.}

2.a. Construction of the Approximate Solution. $\Omega$ will denote a simply connected smooth convex and bounded open set of $\mathbf{R}^{2}$. In this open set we consider the solution of the Euler equation

$$
\begin{gathered}
\left.\frac{\partial u}{\partial t}+u \cdot \nabla u=-\nabla p, \quad \nabla \cdot u=0 \quad \text { in } \Omega \times\right] 0, T[, \\
\left.u(x, t) \cdot n(x)\right|_{\partial \Omega}=0 .
\end{gathered}
$$

$n(x)$ denotes the outward normal to $\partial \Omega$, boundary of $\Omega$ and (2.2) means that the fluid is tangent to the boundary of $\Omega$. Nonhomogeneous boundary conditions can be handled but we restrict ourselves to this case for clarity. When the initial data $u_{0}(x)$ or $\omega_{0}(x)=\nabla \Lambda u_{0}(x)$ are known the solution of (2.1) and (2.2) is completely determined. Indeed using the stream function $\psi$ or the vorticity $\omega=\nabla \Lambda u$, one can show that (2.1) and (2.2) are equivalent to the equations

$$
\begin{gathered}
\frac{\partial \omega}{\partial t}+u \nabla \omega=0 \quad \text { in } \Omega \times \mathbf{R}_{t} \\
u=\nabla \Lambda \psi=\left(\frac{\partial \psi}{\partial x_{2}},-\frac{\partial \psi}{\partial x_{1}}\right) \\
-\Delta \psi=\omega,\left.\quad \psi\right|_{\partial \Omega}=0 .
\end{gathered}
$$

We will introduce the $\mathrm{P}^{1}$ approximation of (2.4) and (2.5). We will denote by $\Omega_{h}$ a convex polygonal approximation of $\Omega$, constructed with a regular triangulation $\mathcal{T}_{h}$ 
as in 1.b. We will assume that the boundary vertices of $\mathscr{T}_{h}$ belong to $\partial \Omega_{h} \cap \partial \Omega$. We will denote by $V_{h}$ the space of continuous functions on $\Omega_{h}$ which are linear affine on each triangle $\mathcal{T}_{h}$ and which vanish on the boundary $\partial \Omega_{h}$.

The Discretized Problem. $u_{h}(x, t)$ is chosen constant on every prism $P_{i k}=\tau_{i} \times$ $[k \Delta t,(k+1) \Delta t]$; it is defined by the relations

$$
\begin{gathered}
u_{h}(x, t)=\nabla \Lambda \psi_{h}^{k} \quad \text { for } t \in[k \Delta t,(k+1) \Delta t[, \\
\psi_{h}^{k} \in V_{h} \text { and } \int_{\Omega_{h}} \nabla \psi_{h}^{k} \nabla \theta_{h} d x=\sum_{\tau_{i} \in \sigma_{h}} \omega_{h}\left(\xi^{i k}, k \Delta t\right) \int_{\tau_{i}} \theta_{h}(x) d x, \quad \forall \theta_{h} \in V_{h} .
\end{gathered}
$$

$\omega_{h}$ satisfies the transport equation

$$
\frac{\partial \omega_{h}}{\partial t}+u_{h} \nabla \omega_{h}=0, \quad \omega(x, 0)=\left(\nabla \Lambda u_{0}\right)(x)
$$

and $\xi^{i k} \in P_{i k}$ are chosen according to Algorithm 4 which constructs an exact solution of (2.6), (2.8).

Algorithm 4: Construct a solution of (2.6), (2.8).

0 . Choose $\xi^{i 0} \in \tau_{i}, i=1, \ldots, N$. Set $t^{0}=0, k=0$.

Set $\omega_{h}\left(\xi^{i 0}, 0\right)=\nabla \Lambda u_{0}\left(\xi^{i 0}\right)$.

1. For $i=0$ to $N$ do

compute the solution of (2.6), (2.7)

with Procedure $F\left(\xi^{i k}, t^{k}, \Delta t, u_{h}, 1\right)$ compute all the nodes of $\left\{x_{m}^{i k}, t_{m}^{i k}\right\}_{1}^{m_{i k}}$ of the characteristic solution of

$$
\left.\left.\frac{d X}{d \tau}=u_{h}(X, \tau) \text { for all } \tau \in\right] t^{k}, t^{k}+\Delta t\right] \text { with } X\left(t^{k}\right)=\xi^{i k} .
$$

Set

$$
\xi^{j(i), k+1}=x_{m_{i k}}^{i k}, \quad t^{k+1}=t^{k}+\Delta t, \quad \omega_{h}\left(\xi^{j(i), k+1}, t^{k+1}\right)=\omega_{h}\left(\xi^{i k}, t^{k}\right),
$$

where $j(i)$ is such that $\xi^{j(i), k+1} \in P_{j(i), k+1}$.

2. For all $i^{\prime} \leqslant N$ such that $j(i) \neq i^{\prime}, \forall i$ do with Procedure $F\left(\xi^{i^{\prime} 0}, t^{k+1}, t^{k+1},-u_{h},-1\right)$ compute all the nodes $\left\{x_{m}^{i k}, t_{m}^{k}\right\}_{1}^{m_{i k}}$ of the characteristic solution of (2.9) for all $\tau \in] 0, t^{k+1}\left[\right.$ with $X\left(t^{k+1}\right)=\xi^{i^{\prime \prime} 0}$, and set $\xi^{i^{\prime}, k+1}=\xi^{i^{\prime}, 0}, \omega_{h}\left(\xi^{i^{\prime}, k+1}, t^{k+1}\right)=\omega_{h}\left(\xi^{l, 0}\right)$ where $l$ is such that $X(0) \in$ $P_{l, 0}$.

Remark 1. Algorithm 4 proceeds exactly like Algorithm 2 to integrate (2.8). The fact that $u_{h}$ depends upon $\omega_{h}$ is not a problem because $\omega_{h}$ can be computed on $[k \Delta t,(k+1) \Delta t]$ if $u_{h}$ is known on $[0, k \Delta t]$.

Remark 2. As before one could choose a $\theta$ and compute the characteristics backward on a time interval $\theta$ only.

2.2. Error Bounds. Equation (2.8) is a transport equation; therefore the sharpest a priori bound for the solution $u_{h}, \omega_{h}$ is given by the $L^{\infty}$ norm of $\omega_{h}$. Namely we have

$$
\left|\omega_{h}(\cdot, t)\right|_{L^{\infty}\left(\Omega_{h}\right)} \leqslant\left|\omega_{0}(\cdot)\right|_{L^{\infty}(\Omega)} \text {. }
$$

The situation is similar when one tries to prove the regularity of the exact solution of the Euler equation in two dimensions. From the relation (2.10) one deduces that $\omega$ is bounded uniformly in $L^{\infty}(\Omega)$. Therefore the solution of the elliptic system

$$
\nabla \Lambda u=\omega, \quad \nabla \cdot u=0,\left.\quad u \cdot \nu\right|_{\partial \Omega}=0
$$


is uniformly bounded in $L^{\infty}\left(\mathbf{R}^{+} ; W^{1, p}(\Omega)\right)$ for $1<p<\infty$ but not for $p=\infty$, and it is not possible to prove directly that the higher order derivatives of $u$ remain bounded. This fact is related to the nature of the Green's function of the Laplace equation: from the solution of the equation

$$
-\Delta \Psi=\omega,\left.\quad \Psi\right|_{\partial \Omega}=0,
$$

$u$ is given by the formula

$$
u=\nabla \Lambda \Psi=\left(\frac{\partial \Psi}{\partial x_{2}},-\frac{\partial \Psi}{\partial x_{1}}\right)
$$

and is not Lipschitzian, but satisfies the following a priori estimate

$$
|u(x)-u(y)| \leqslant C|x-y| \log \frac{D}{|x-y|}|\omega|_{\infty}
$$

where $D$ denotes the diameter of the open set $\Omega$. Now let $x(t)$ and $y(t)$ be the trajectories of two particles of the fluid and denote by $\rho(t)=|x(t)-y(t)|$ the Euclidean distance between $x(t)$ and $y(t)$. Since $x(t)$ and $y(t)$ are the solutions of the differential equations

$$
\dot{x}(t)=u(x(t), t), \quad \dot{y}(t)=u(y(t), t),
$$

one deduces from (2.11) the a priori estimates

$$
|\dot{\rho}(t)|=|u(x(t), t)-u(y(t), t)| \leqslant C \rho \log \frac{D}{\rho}|\omega|_{\infty} .
$$

By a comparison argument one finally obtains

$$
\left(\frac{\rho(0)}{D}\right)^{e^{c|\omega| \infty t}} \leqslant \frac{\rho(t)}{D} \leqslant\left(\frac{\rho(0)}{D}\right)^{e^{-C|\omega| \infty \infty^{t}}} .
$$

The first inequality of (2.14) is the cornerstone of the proof of the regularity of $\omega$ (in $C^{0, \alpha}(\Omega)$ ). Then one obtains easily that $u$ is bounded in $C^{1, \alpha}$ (see Wolibner [14], Schaeffer [13] and Kato [11] for details), but the bounds involve constants, like those in (2.14), rapidly growing with $t$. Nevertheless, for smooth data we have

$$
u \in W^{1, \infty}(Q) \text {. }
$$

To get an error estimate on $\left|\omega-\omega_{h}\right|_{\infty}$ we shall need the following lemmas:

LEMMA 1 (TARTAR). If $0<\alpha<1$ and if

$$
\begin{gathered}
\left.\left|\frac{d}{d t} \delta(\tau)\right| \leqslant c_{4} h^{1-\alpha}+c_{5} \delta(0)+c_{6} \delta(\tau) \quad \forall \tau \in\right] 0, t[, \\
\delta(t) \leqslant c_{7}(h+\Delta t),
\end{gathered}
$$

then

$$
\left.\delta(\tau) \leqslant c_{8}\left(h^{1-\alpha}+\Delta t\right) \quad \forall \tau \in\right] 0, t[.
$$

Proof. Note first of all that if (2.15) were an equality then

$$
\begin{aligned}
\delta(\tau)= & {\left[\frac{c_{4}}{c_{6}} h^{1-\alpha}+\frac{c_{5}}{c_{6}} \frac{c_{7}(h+\Delta t) c_{6} \varepsilon e^{-c_{6} e t}+c_{4} h^{1-\alpha}\left(e^{-c_{6} e t}-1\right)}{c_{6} \varepsilon-c_{5}\left(e^{-c_{6} e t}-1\right)}\right] } \\
& \cdot\left(e^{c_{6} e(\tau-t)}-1\right)+c_{7}(h+\Delta t) e^{c_{6} e(\tau-t)}
\end{aligned}
$$


with $\varepsilon= \pm 1$; therefore the result is reasonable. Now to prove it one has to use the Bellman-Gronwall lemma on time intervals of length $\theta$ such that

$$
\theta \leqslant \frac{1}{c_{6}} \log \left(1+1 / 2 c_{5}\right) \text {. }
$$

Indeed on $] 0, \theta[(2.15)$ implies

$$
\delta(0)\left[1-c_{5}\left(e^{c_{6} \theta}-1\right) / c_{6}\right] \leqslant \delta(\theta) e^{c_{6} \theta}+\left(e^{c_{6} \theta}-1\right) c_{4} h^{1-\alpha} / c_{6}
$$

and (2.18) yields then

$$
\delta(0) \leqslant 2 c_{6} e^{c_{6} \theta} \delta(\theta)+2\left(e^{c_{6} \theta}-1\right) c_{4} h^{1-\alpha} .
$$

In turn (2.15) and (2.20) imply

$$
\left|\frac{d}{d \tau} \delta(\tau)\right| \leqslant c_{4} h^{1-\alpha}\left[1+2\left(e^{c_{6} \theta}-1\right) c_{5}\right]+2 c_{6} c_{5} e^{c_{6} \theta} \delta(\theta)+c_{6} \delta(\tau) .
$$

So by repeating the same argument on $\tau \in] \theta, 2 \theta[$ and so on one finds eventually

$$
\left|\frac{d}{d \tau} \tau(\tau)\right| \leqslant c_{9} h^{1-\alpha}+c_{10}(h+\Delta t)+c_{6} \delta(\tau)
$$

which yields (2.16).

Lemma 2. Assume that $\Omega$ is a bounded convex open set, let $T_{h}$ denote a regular triangulation of $\Omega$. We will assume that $\Omega_{h}=U\left\{k: k \in T_{h}\right\}$ is contained in $\Omega$ and that every interval belonging to $\partial \Omega_{h}$ has its vertices on $\partial \Omega$. As usual we will assume that the diameter of $k$ is of the order of $h$ and that there exists a constant $C$ independent of $h$ and $k_{h}$ so that one has, for every triangle $k_{h} \in T_{h}$ :

(diameter of $\left.k_{h}\right) \leqslant C$ (diameter of the circle inscribed in $\left.k_{h}\right)$.

For every function $\omega \varepsilon L^{\infty}(\Omega)$, we introduce the functions $\psi$ and $\psi_{h}$ by the relations

$$
\begin{gathered}
\psi \in H_{0}^{1}(\Omega), \quad \int_{\Omega} \nabla \Psi \nabla \theta=\int_{\Omega} \omega \theta d x \quad \forall \theta \in H_{0}^{1}(\Omega), \\
\psi_{h} \in V_{h}, \quad \int_{\Omega_{h}} \nabla \psi_{h} \nabla \theta_{h}=\int_{\Omega_{h}} \omega \theta_{h} d x \quad \forall \theta_{h} \in V_{h} .
\end{gathered}
$$

Then we have for any $p, 1 \leqslant p<\infty$,

$$
\left|\psi-\psi_{h}\right|_{W^{1, \infty}(\Omega)} \leqslant C h^{1-1 / p}|\omega|_{L^{\infty}(\Omega)} .
$$

In (2.26) $C$ denotes a constant independent of $h$ and $\omega$.

Proof. The proof follows with slight modifications the line of the proof given by Nitsche [12] which is also described in the book of Ciarlet [4]. First we notice that any $\theta_{h} \in V_{h}$ can be extended by zero in $\Omega-\Omega_{h}$ and defines a function still denoted by $\theta_{h}$ which belongs to $H_{0}^{1}(\Omega)$. Therefore $V_{h}$ is a closed subspace of $H_{0}^{1}(\Omega)$. Conversely let $\Pi_{h} \psi$ be the $P_{1}$ Lagrange interpolate of any function $\psi \in H_{0}^{1}(\Omega) \cap$ $H^{2}(\Omega)$, ( $\psi$ being continuous, this expression makes sense). $\Pi_{h} \psi$ vanishes on $\partial \Omega_{h}$; therefore $\Pi_{h} \psi \in V_{h}$ and we have (cf. Ciarlet [4, (3.1.39), Chapter 3, §3.1, p. 123]):

$$
\left|\psi-\Pi_{h} \psi\right|_{W^{k, \infty}\left(\Omega_{h}\right)} \leqslant C h^{2-k-2 / p}|\psi|_{W^{2 \rho}\left(\Omega_{h}\right)}, \quad k=0 \text { or } 1 \text { and } p>2 .
$$

Using (2.24) and the fact that $\omega$ is in $L^{\infty}(\Omega)$, we deduce from(2.27) that we have for 
$k=0,1$

$$
\left|\psi-\Pi_{h} \psi\right|_{W^{k, \infty}\left(\Omega_{h}\right)} \leqslant C h^{2-k-2 / p}|\omega|_{L^{\infty}(\Omega)} .
$$

(As usual (2.26) is valid only for $p<+\infty$.) Next on the space $H_{0}^{1}\left(\Omega_{h}\right) \cap W^{1, \infty}\left(\Omega_{h}\right)$ and on the space $V_{h}$ we will use the weighted norms

$$
v \rightarrow|v|_{0, \infty, \Omega}+h|\log h||v|_{1, \infty, \Omega}
$$

and

$$
v \rightarrow|\log h|^{-1 / 2}|v|_{0, \infty, \Omega}+h|v|_{1, \infty, \Omega} .
$$

From (2.25) we deduce that $\psi_{h}$ is the projection of $\psi: P_{h} \psi$ (for the scalar product $\left.\int_{\Omega} \nabla u \nabla v d x\right)$ on $V_{h}$. Therefore we have (Ciarlet [4, (3.3.61), p. 163]),

$$
|\log h|^{-1 / 2}\left|P_{h} \psi\right|_{0, \infty, \Omega_{h}}+h\left|P_{h} \psi\right|_{1, \infty, \Omega_{h}} \leqslant c\left(|\psi|_{0, \infty, \Omega_{h}}+h|\log h||\psi|_{1, \infty, \Omega_{h}}\right)
$$

Next we have

$$
\begin{aligned}
|\log h|^{-1 / 2} \mid \psi- & \left.\psi_{h}\right|_{0, \infty, \Omega_{h}}+h\left|\psi-\psi_{h}\right|_{1, \infty, \Omega_{h}} \\
& =|\log h|^{-1 / 2}\left|\left(I-P_{h}\right) \psi\right|_{0, \infty, \Omega_{h}}+h\left|\psi-\psi_{h}\right|_{1, \infty, \Omega_{h}} \\
& =|\log h|^{-1 / 2}\left|\left(I-P_{h}\right)\left(\psi-v_{h}\right)\right|_{0, \infty, \Omega_{h}}+h\left|\psi-\psi_{h}\right|_{1, \infty, \Omega_{h}} \\
& \leqslant C\left(\left|\psi-v_{h}\right|_{0, \infty, \Omega_{h}}+h|\log h|\left|\psi-v_{h}\right|_{1, \infty, \Omega_{h}}\right)
\end{aligned}
$$

where $C$ denotes a constant independent of $h$ for $h$ small enough, and $v_{h}$ any element of $V_{h}$. Now taking for $v_{h}$ the interpolate $\Pi_{h} \psi$ and using (2.27) we obtain

$$
\begin{aligned}
&\left|\psi-\psi_{h}\right|_{0, \infty, \Omega_{h}}+h|\log h|^{-1 / 2}\left|\psi-\psi_{h}\right|_{1, \infty, \Omega_{h}} \\
& \leqslant\left(|\log h|^{-1 / 2} h^{2-2 / p}+h|\log h|^{3 / 2} h^{1-2 / p}\right)|\psi|_{W^{2 \rho}\left(\Omega_{h}\right)}
\end{aligned}
$$

From (2.28) we also have

$$
|\psi|_{2, p, \Omega} \leqslant C|\omega|_{L^{\infty}(\Omega)}
$$

These estimates remain valid for domain $\Omega$ with corners of angle $\mathcal{\theta}_{i}$, provided one has

$$
\max \left(\Pi / \vartheta_{i}\right)<p
$$

With the relation (2.21) and Lemma 2.1 we can give an error estimate:

Theorem 3. Assume that $\Omega$ is a smooth regular convex open set of $\mathbf{R}^{2}$ and that the initial data $u_{0}(\cdot)$ is a smooth function $\left(u_{0}(\cdot)\right.$ belongs to $C^{2}(\Omega)$ for instance); then the solution of $\left(P_{h}\right)$ : (2.6), (2.7) and (2.8) converges to the solution of (2.1), (2.2) (or equivalently to the solution of (2.3), (2.4), (2.5)). More precisely, denoting by $\Delta t$ the time step and by $h$ the parameter of the triangulation, we have

$$
\begin{aligned}
& \forall \varepsilon \in] 0,1\left[\text { there exists } h^{0}, \Delta t^{0}\right. \text { such that: } \\
& \left|\omega_{h}(x, t)-\omega(x, t)\right| \leqslant C(t)\left(h^{1-e}+\Delta t\right) \quad \forall h \leqslant h^{0}, \Delta t \leqslant \Delta t^{0} .
\end{aligned}
$$

Remark. In (2.34) $C(t)$ denotes a constant depending on $u_{0}$ and $t$, but not on $h$, $\Delta t$, furthermore $C(t)$ is rapidly growing towards $C(T)$ when $t$ increases. As we mentioned in the beginning of the section $C(T)$ may itself grow very fast with $T$. Nevertheless, Theorem 3 shows that the method is of order $h^{1-\varepsilon}+\Delta t$. 
Proof of Theorem 3. By construction

$$
\begin{aligned}
\left|\omega_{h}(x, t)-\omega(x, t)\right| & \leqslant\left|\omega_{0 h}\left(X_{h}^{\xi^{i j, t} t^{j}}(0)\right)-\omega_{0}\left(X^{x, t}(0)\right)\right| \\
& \leqslant\left|\omega_{0 h}-\omega_{0}\right|_{\infty}+\left|\nabla \omega_{0}\right|_{\infty}\left|X_{h}^{\xi^{j,}, t^{j}}(0)-X^{x, t}(0)\right|,
\end{aligned}
$$

where the second inequality was obtained from the first by adding and subtracting $\omega_{0}\left(X_{h}^{\xi^{i j}, t^{j}}(0)\right)$ in the first one. Therefore

$$
\left|\omega_{h}(\cdot, t)-\omega(\cdot, t)\right|_{\infty} \leqslant C_{11} h+\left|\nabla \omega_{0}\right|_{\infty} \sup _{x, t}\left|X_{h}^{\xi^{j j}(x), t^{j}}(0)-X^{x, t}(0)\right| .
$$

Now by construction of $X_{h}$ and $X$ we have

$$
\begin{aligned}
\left|\frac{d}{d \tau}\left(X_{h}^{\xi^{i j}, t^{j}}(\tau)-X^{x, t}(\tau)\right)\right| & =\left|u_{h}\left(X_{h}^{\xi^{i j}, t^{j}}(\tau), \tau\right)-u\left(X^{x, t}(\tau), \tau\right)\right| \\
& \leqslant\left|u_{h}(\cdot, \tau)-u(\cdot, \tau)\right|_{\infty}+|\nabla u|_{\infty}\left|X_{h}^{\xi^{i j}, t^{j}}(\tau)-X^{x, t}(\tau)\right|
\end{aligned}
$$

(again to get the last inequality one adds and subtracts $u\left(X_{h}^{\xi^{i}, t^{j}}(\tau), \tau\right)$ ). Let $\tilde{\psi}$ be the solution of

$$
-\Delta \tilde{\psi}=\omega_{h} \quad \text { in } \Omega,\left.\quad \tilde{\psi}\right|_{\Gamma}=0 .
$$

Then, according to Lemma 2 ,

$$
\begin{aligned}
\left|u_{h}(\cdot, \tau)-u(\cdot, \tau)\right|_{\infty} \leqslant & \left|\nabla \Lambda \psi_{h}(\cdot, t)-\nabla \Lambda \tilde{\psi}(\cdot, t)\right|_{\infty} \\
& +|\nabla \Lambda \tilde{\psi}(\cdot, t)-\nabla \Lambda \psi(\cdot, t)|_{\infty} \\
\leqslant & C_{12} h^{1-\varepsilon}\left|\omega_{h}(\cdot, t)\right|_{\infty}+C_{13}\left|\omega_{h}(\cdot, t)-\omega(\cdot, t)\right|_{\infty}
\end{aligned}
$$

the last term comes from the continuity of $(-\Delta)^{-1}$ from $L^{\infty}(\Omega)$ into $W^{1, \infty}(\Omega)$. Now (2.35), (2.37) and (2.39) yield

$$
\begin{aligned}
& \left|\frac{d}{d \tau}\left(X_{h}^{\xi^{j}, t^{j}}(\tau)-X_{h}^{x, t}(\tau)\right)\right| \leqslant C_{12}\left|\omega_{h}(\cdot, t)\right|_{\infty} h^{1-\varepsilon} \\
& \quad+C_{13}\left(\left|\omega_{0 h}-\omega_{0}\right|_{\infty}+\left|\nabla \omega_{0}\right|_{\infty} \sup _{x, t}\left|X_{h}^{\xi^{i j}(x), t^{j}(t)}(0)-X^{x, t}(0)\right|\right) \\
& \quad+|\nabla u|_{\infty}\left|X_{h}^{\xi^{j,}, t^{j}}(\tau)-X^{x, t}(\tau)\right| .
\end{aligned}
$$

If we take $x=x^{*}, t=t^{*}$ where the ${ }^{*}$ denotes the values for which the sup in (2.40) is attained, then Lemma 1 gives

$$
\left|X_{h}^{\xi^{i j}\left(x^{*}\right), t^{j}\left(t^{*}\right)}(0)-X^{x^{*}, t^{*}}(0)\right| \leqslant C_{14}\left(h^{1-\varepsilon}+\Delta t\right),
$$

and finally the Bellman-Gronwall lemma yields the result.

\section{Preliminary Numerical Experiments.}

Test 1 . We have solved problem (2.3), (2.5), with

$$
\Omega=] 0,1\left[2, \quad \omega_{0}=\left[\begin{array}{lr}
10 & \text { in }[.22, .33 \\
1 & \text { elsewhere, }
\end{array} \times[.11, .22],\right.\right.
$$

and we have triangulated $\Omega$ with 3 families of parallel lines $h=1 / 9, \Delta t=.1$. At later times $\omega$ can only be equal to 10 or 1 . Therefore the initial spot, $\omega=10$, is expected to turn with the flow and the area of $\{x: \omega(x, t)=10\}$ remains constant. 
Since our scheme is conservative in a statistical sense only the area mentioned above is not constant, but it fluctuates around the exact value. The spot can be seen to turn with the flow.

Test 2. Similar problem in a ring. These problems are particularly difficult for dissipative numerical methods.

Test 3. This test simulates the dispersion of a pollutant (chimney smoke) by a wind. The pollutant comes out of $\Gamma_{1}$, a part of the boundary $\Gamma$, with initial velocity 2.7 times the initial velocity of the wind and a vorticity of 10 , while the wind has no initial vorticity; $\Omega$ contains a hump (hill) to illustrate the feasibility of the finite element method.
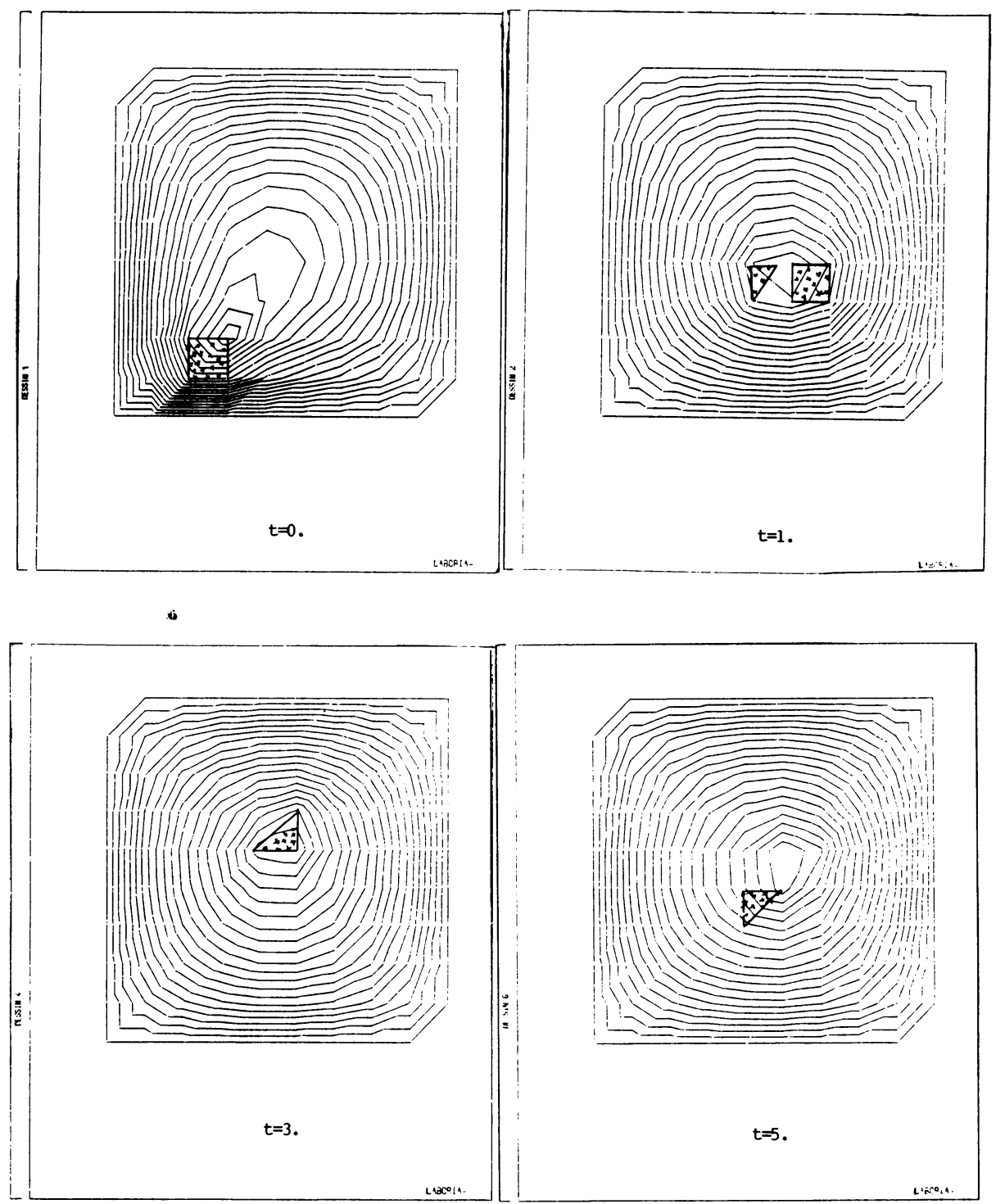

Figure 1 

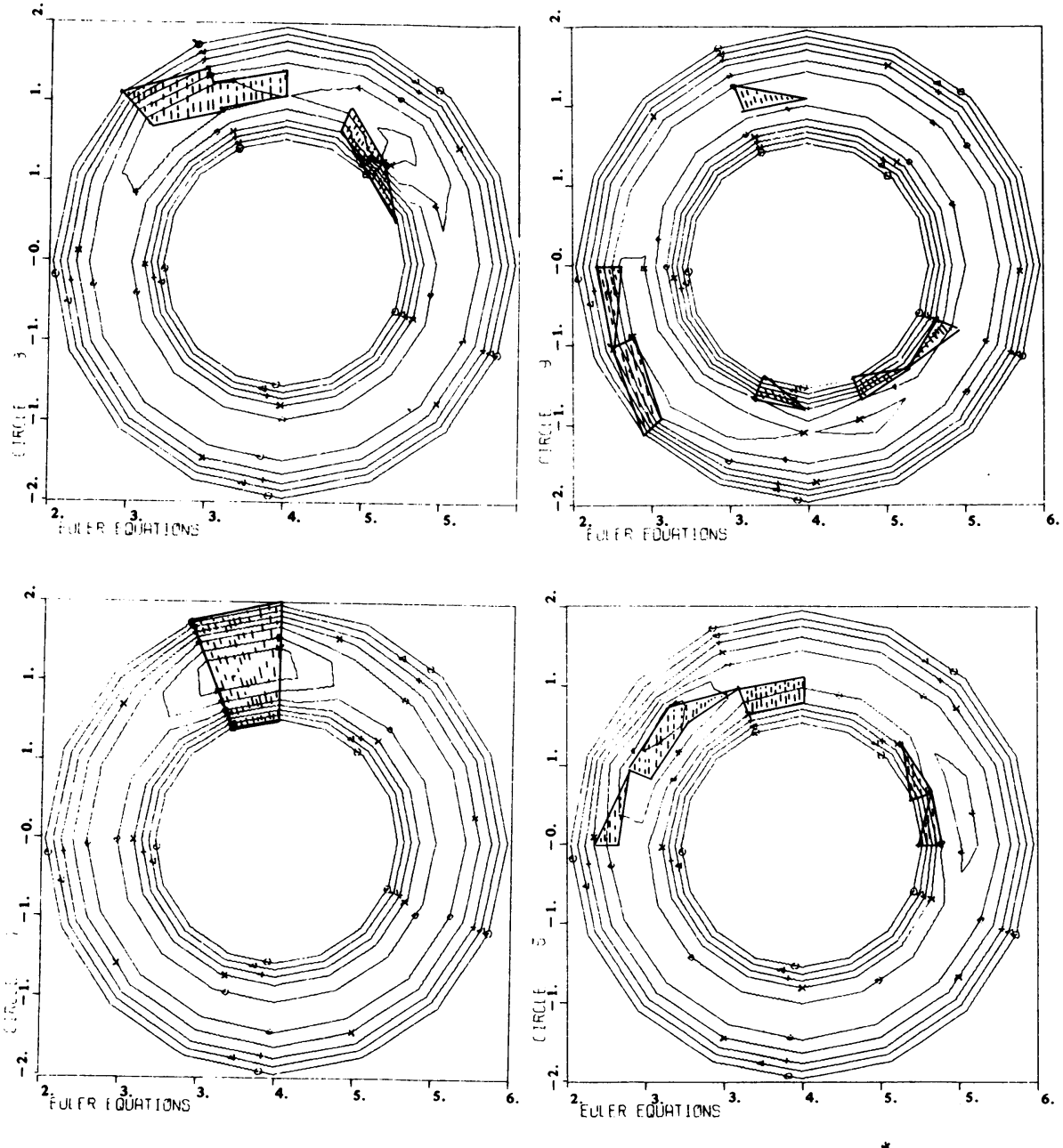

FIGURE 2

Therefore we have solved

$$
\begin{gathered}
\frac{\partial \omega}{\partial t}+(\nabla \Lambda \Psi) \nabla \omega=0, \quad \omega(t=0)=0 \\
\left.\omega\right|_{\Gamma_{1}}=10,\left.\quad \omega\right|_{\Gamma_{2}}=0, \quad-\Delta \Psi=\omega \\
\left.\frac{\partial \Psi}{\partial y}\right|_{\Gamma_{2} \cup \Gamma_{3}}=1, \quad-\left.\frac{\partial \Psi}{\partial x}\right|_{\Gamma_{1}}=2.7,\left.\quad \Psi\right|_{\Gamma_{4}}=\text { constant. }
\end{gathered}
$$

The triangulation shows 225 nodes and $\Delta t=.2$.

Computing times are 30" on an IBM 370. 168 for Tests 1 and 2 and $1 \mathrm{mn}$ for Test 3. The plottings of the vorticity (shaded area on the figures) are done by hand because it is piecewise constant on the triangulation. 

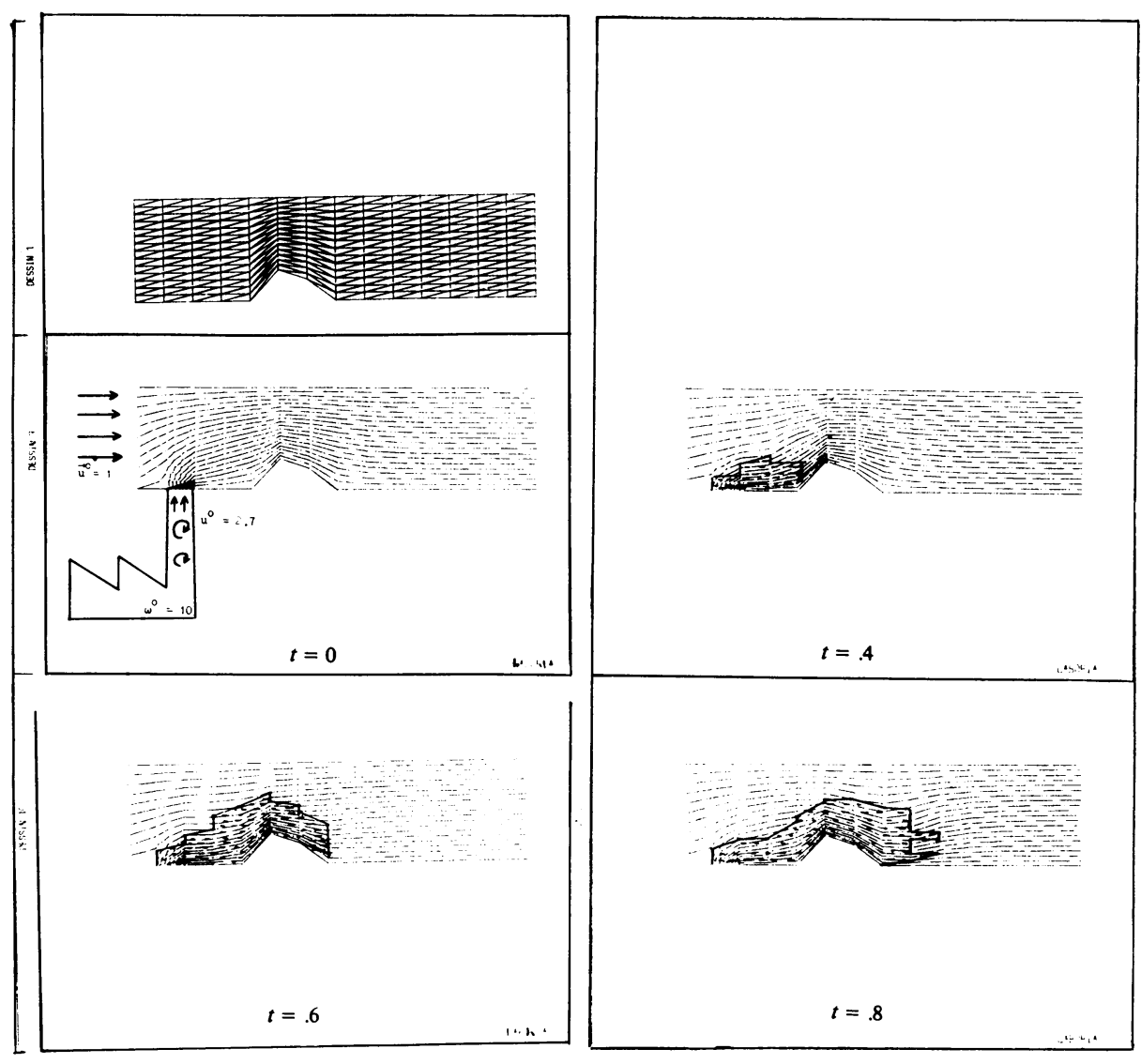

FigURE 3

Conclusion. This work gives some results on the implementation of the method of characteristics in a finite element context. Error estimates and applications to the vortex method are given. Practical applications however will involve shocks and/or boundary layers if there is a slight dissipation. Extension of these results to include dissipation is in progress.

\section{Department of Mathematics \\ University of Paris 13 \\ CSP-93430 Villetaneuse, France}

School of Applied Sciences

University of Jerusalem

Givat Ram, Israel

Department of Mathematics

University of Paris 13

CSP-93430 Villetaneuse, France

and

INRIA

Domaine de Voluceau

Rocquencourt

B. P. 10578150 , Lechesnay, France 
1. V. ARnold, Problèmes Ergotiques de la Mécanique Classique, Gauthier-Villars, Paris, 1967.

2. G. BAKER, "The clouds and cells technique applied to the roll up of vortex sheets," J. Comput. Phys., 1979, pp. 75-95.

3. C. BARDos, "Existence et unicité de la solution de l'équation d'Euler en dimension deux," J. Math. Anal. Appl., v. 40, 1972, pp. 769-790.

4. Ph. Cinrlet, The Finite Element Method for Elliptic Problems, Studies in Math. and Its Applications, North-Holland, Amsterdam, 1978.

5. A. J. Chorin, "Numerical study of slightly viscous flow," J. Fluid Mech., v. 57, 1973, pp. 784-796.

6. J. P. Christiansen, "Numerical solution of hydrodynamics by the method of point vortices," $J$. Comput. Phys., v. 13, 1973, pp. 863-879.

7. B. Couet, O. Buneman \& A. LeOnard, Three Dimensional Simulation of the Free Shear Layer Using the Vortex in Cell Method (Proc. 2nd Sympos. on Turbulent Shear Flows, London, 1979).

8. P. GRISVARD, "Behaviour of the solution of an elliptic boundary value problem in a polygonal or polyhedral domain," Numerical Solutions of Differential Equations, vol. 3 (B. Hubbard, Ed.), Academic Press, New York, 1976, pp. 267-274.

9. M. O. HALD, “Convergence of vortex methods for Euler's equations," SIAM J. Numer. Anal., v. 16, 1979, pp. 726-755.

10. M. O. Hald \& V. Mauceri Del Prete, "Convergence of vortex methods for Euler's equations," Math. Comp., v. 32, 1978, pp. 791-809.

11. T. KAтo, "On the classical solution of the two dimensional nonstationary Euler equation," Arch. Rational Mech. Anal., v. 25, 1967, pp. 188-200.

12. J. NitSCHE, “ $L_{\infty}$ convergence of finite element approximation," Mathematical Aspects of Finite Element Methods, Springer, Berlin and New York, 1975.

13. A. C. SCHAEFFER, "Existence theorem for the flow of an incompressible fluid in two-dimension," Trans. Amer. Math. Soc., v. 42, 1967, p. 497.

14. W. WOLIBNER, "Un théorème sur l'existence du mouvement plan d'un fluide parfait homogène et incompressible pendant un temps infiniment long," Math. Z., v. 37, 1935, pp. 727-738.

15. M. ZeRner, "Equations d'évolution quasi-linéaire du premier ordre: Le cas lipschitzien." ( $\dot{A}$ paraître.) 